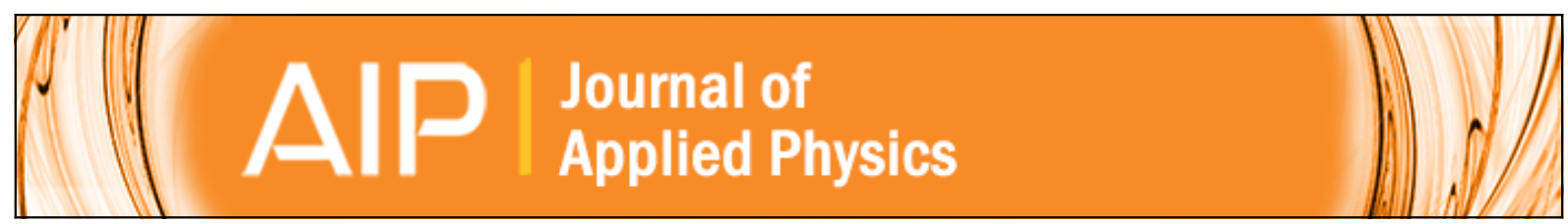

Controlled route to the fabrication of carbon and boron nitride nanoscrolls: A molecular dynamics investigation

Eric Perim, Ricardo Paupitz, and Douglas S. Galvão

Citation: Journal of Applied Physics 113, 054306 (2013); doi: 10.1063/1.4790304

View online: http://dx.doi.org/10.1063/1.4790304

View Table of Contents: http://scitation.aip.org/content/aip/journal/jap/113/5?ver=pdfcov

Published by the AIP Publishing

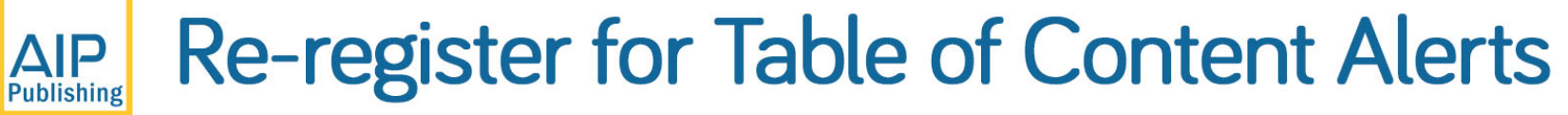

Create a profile.

Sign up today! 


\title{
Controlled route to the fabrication of carbon and boron nitride nanoscrolls: A molecular dynamics investigation
}

\author{
Eric Perim, ${ }^{1}$ Ricardo Paupitz, ${ }^{2, a)}$ and Douglas S. Galvão ${ }^{1}$ \\ ${ }^{1}$ Instituto de Física "Gleb Wataghin," Universidade Estadual de Campinas, Unicamp, C.P. 6165 , \\ 13083-970 Campinas, São Paulo, Brazil \\ ${ }^{2}$ Departamento de Física, IGCE, Univ Estadual Paulista, UNESP, 13506-900 Rio Claro, SP, Brazil
}

(Received 14 November 2012; accepted 15 January 2013; published online 6 February 2013)

\begin{abstract}
Carbon nanoscrolls (graphene layers rolled up into papyrus-like tubular structures) are nanostructures with unique and interesting characteristics that could be exploited to build several new nanodevices. However, an efficient and controlled synthesis of these structures was not achieved yet, making its large scale production a challenge to materials scientists. Also, the formation process and detailed mechanisms that occur during its synthesis are not completely known. In this work, using fully atomistic molecular dynamics simulations, we discuss a possible route to nanoscrolls made from graphene layers deposited over silicon oxide substrates containing chambers/pits. The scrolling mechanism is triggered by carbon nanotubes deposited on the layers. The process is completely general and can be used to produce scrolls from other lamellar materials, like boron nitride, for instance. (C) 2013 American Institute of Physics. [http://dx.doi.org/10.1063/1.4790304]
\end{abstract}

\section{INTRODUCTION}

Carbon nanoscrolls (CNSs) are structures which have been known for many decades, ${ }^{1}$ but unlike their closely related structures, the carbon nanotubes $(\mathrm{CNTs}),{ }^{2}$ they have not been as widely studied, despite exhibiting unique and interesting properties. These interesting properties arise from the peculiar topology of CNSs. While CNTs can be viewed as graphene sheets rolled up in a way that its ends meet, forming a cylinder, CNSs can be similarly viewed as rolled up graphene sheets that do not form cylinders, but papyruslike tubular structures. This is better illustrated in Figure 1. The scrolled topology creates flexible diameter structures and present much larger solvent accessible surface than CNTs, since the surface of inner layers can be readily accessed. These unique properties can be exploited to create new potential applications, such as, hydrogen storage ${ }^{3}$ and mechanical actuation. ${ }^{4}$

In spite of these quite appealing properties, there are only few CNS experimental studies. This can be explained, in part, by the difficulties related to its controlled and large scale synthesis. Recently, due to significant advances on the low temperature synthesis techniques of these carbon nanostructures, ${ }^{5,6}$ there is a renewed CNSs interest ${ }^{7-11}$ and some alternative and promising new synthesis routes have been proposed. ${ }^{12-15}$

In principle, using the new approaches, it should be also possible to generate scrolls from other similar lamellar structures, such as boron nitride crystals. Hexagonal BN (hBN) presents the same honeycomb morphology as graphite, with very close bond length values, being known also as the inorganic graphite (or white graphite). Graphene and hBN share many structural and mechanical properties, while presenting a completely different electronic behavior, graphene being a

\footnotetext{
a) Author to whom correspondence should be addressed. Electronic mail: paupitz@rc.unesp.br.
}

semi-metal and hBN a wide bandgap insulator. Because of these structural and mechanical similarities, boron nitride nanoscrolls (BNNSs) have been theoretically predicted, ${ }^{16}$ but they have not been obtained yet in laboratory. As nanotubes and closed cage (fullerene-like) structures have been made out of $\mathrm{BN},{ }^{17,18}$ we can expect that BNNS synthesis can be also feasible.

Recently, Zhang and $\mathrm{Li}^{13}$ reported an investigation on a promising method for synthesizing CNSs, modeled via classical molecular dynamics. In this method, a graphene sheet is deposited over a $\mathrm{SiO}_{2}$ substrate and the scrolling process is triggered by a CNT, in a setup illustrated in Figure 2. Due to the attractive interaction between CNT and graphene sheet, the later can overcome the adhesion to the substrate and then start scrolling. It was shown that this method worked, yielding CNSs, but was very sensitive to the CNT diameter, only working for certain diameter tubes. Despite such limitations, this route could possibly offer good control over the scroll dimensions and easily produces single layered nanoscrolls.

In this work, we further investigate this process, trying to devise new protocols to improve its efficiency and to overcome some of its limitations on tube diameter. We also address how the same principles can be used to produce single layered BNNSs. Similar to Zhang and $\mathrm{Li}^{13}$ approach, we have carried out our study using classical molecular dynamics simulations, as discussed in detail in Sec. II.

\section{METHODOLOGY}

Our system consisted of rectangular pieces of either graphene or boron nitride membranes deposited over a $\mathrm{SiO}_{2}$ substrate and a carbon nanotube which was responsible for triggering the scrolling process, as seen on Figure 2. The graphene (BN) strips had dimensions of $210.5 \AA \times 24.4 \AA$ and the tube had a length of $48 \AA$ with different diameters (from $13.5 \AA$ to $38.0 \AA$ ). 


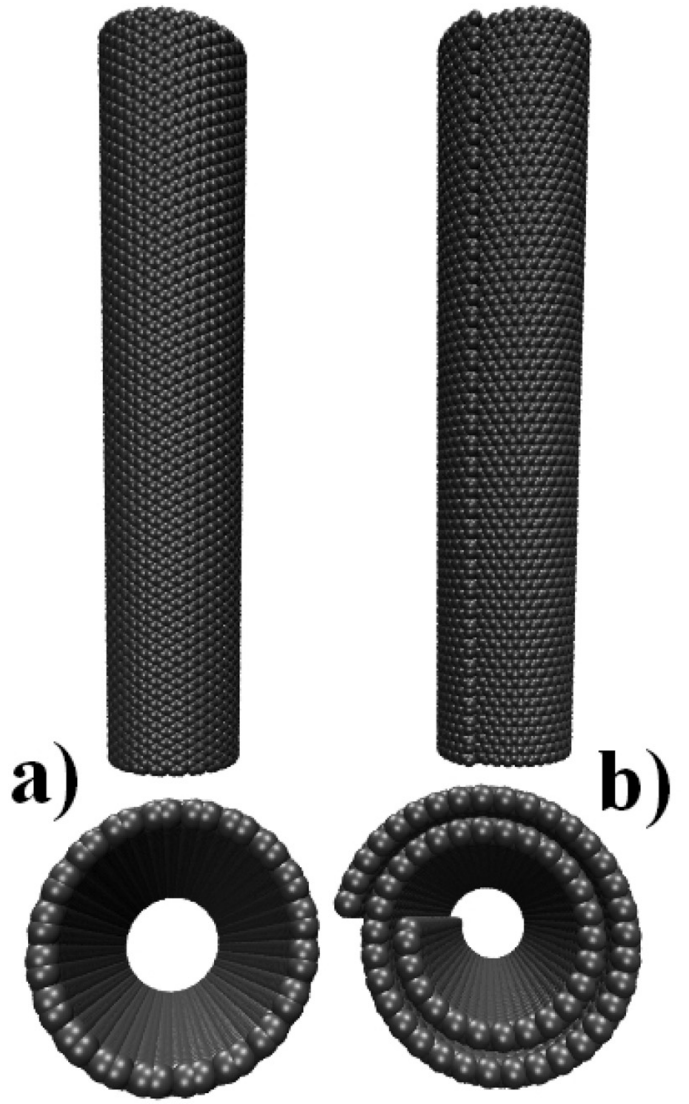

FIG. 1. Lateral and top views of (a) nanotubes and (b) nanoscrolls.

All simulations were carried out using classical molecular dynamics with the universal force field ${ }^{19}$ (UFF), as implemented on the Accelrys MATERIALS STUDIO software. ${ }^{20}$ This force field has been successfully used to study both carbon ${ }^{8}$ and boron nitride ${ }^{16}$ nanoscrolls before. Bond stretching, angle bending, dihedral torsion, and impropers accounted for the bonded interactions, while the non-bonded van de Waals interactions between substrate, sheet, and tube were described by Lennard-Jones potentials

$$
E_{L J}=D_{I J}\left[\left(\frac{r_{I J}}{r}\right)^{12}-2\left(\frac{r_{I J}}{r}\right)^{6}\right]
$$

where $\mathbf{r}$ is the interatomic distance, $\mathbf{D}_{\mathbf{i j}}$ the well depth, and $\mathbf{r}_{\mathbf{i j}}$ is the distance at which the potential reaches its minimum.

The substrate atoms were fixed at all times. Carbon, boron, and nitrogen atoms were assumed neutral and on $\mathrm{sp}^{2}$ hybridization. Geometry optimizations were carried with MATERIALS STUDIO's smart algorithm, ${ }^{20}$ which is a cascade of

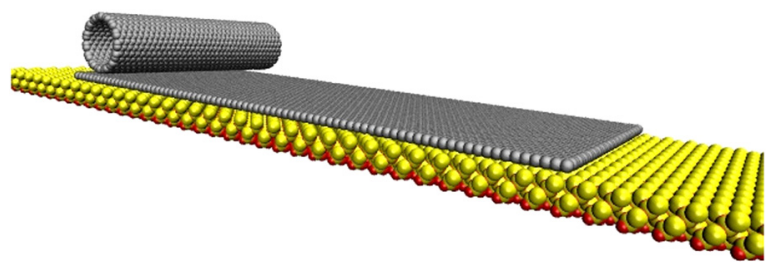

FIG. 2. Carbon nanotube over a graphene membrane deposited on a $\mathrm{SiO}_{2}$ substrate.

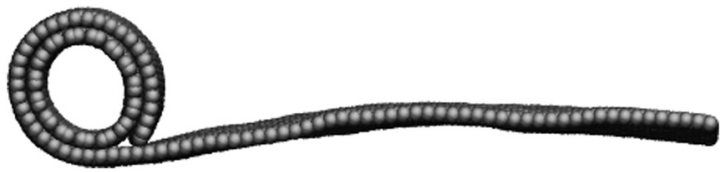

FIG. 3. CNT assisted scrolling of graphene.

the steepest descent, ABNR (adjusted basis set NewtonRaphson), and quasi-Newton methods with tolerance in energy of $2.0 \times 10^{-5} \mathrm{kcal} / \mathrm{mol}$, in force of $0.001 \mathrm{kcalm} / \mathrm{mol} / \AA$, and in displacement of $1.0 \times 10^{-5} \AA$. The dynamics were carried under the NVT, or canonical, ensemble in which the number of particles $(\mathrm{N})$ and the volume $(\mathrm{V})$ of the system are held fixed, while the system has also a well-defined temperature (T). The fixed temperature of $50 \mathrm{~K}$ was controlled using a Nosé thermostat and the integration time step was $1 \mathrm{fs}$.

\section{RESULTS AND DISCUSSIONS}

The principle by which the proposed method is supposed to work is very simple. When a CNT is deposited over a piece of graphene (or $\mathrm{BN}$ ) membrane, the van der Waals interactions lead the sheet to deform itself in order to maximize the contact area and thus the CNT cylindrical morphology leads to the rolling of the sheet, as illustrated in Figure 3 . The process is very efficient when in vacuum since there is no resistance against the elastic forces, but in a more realistic environment, there will be the interaction between
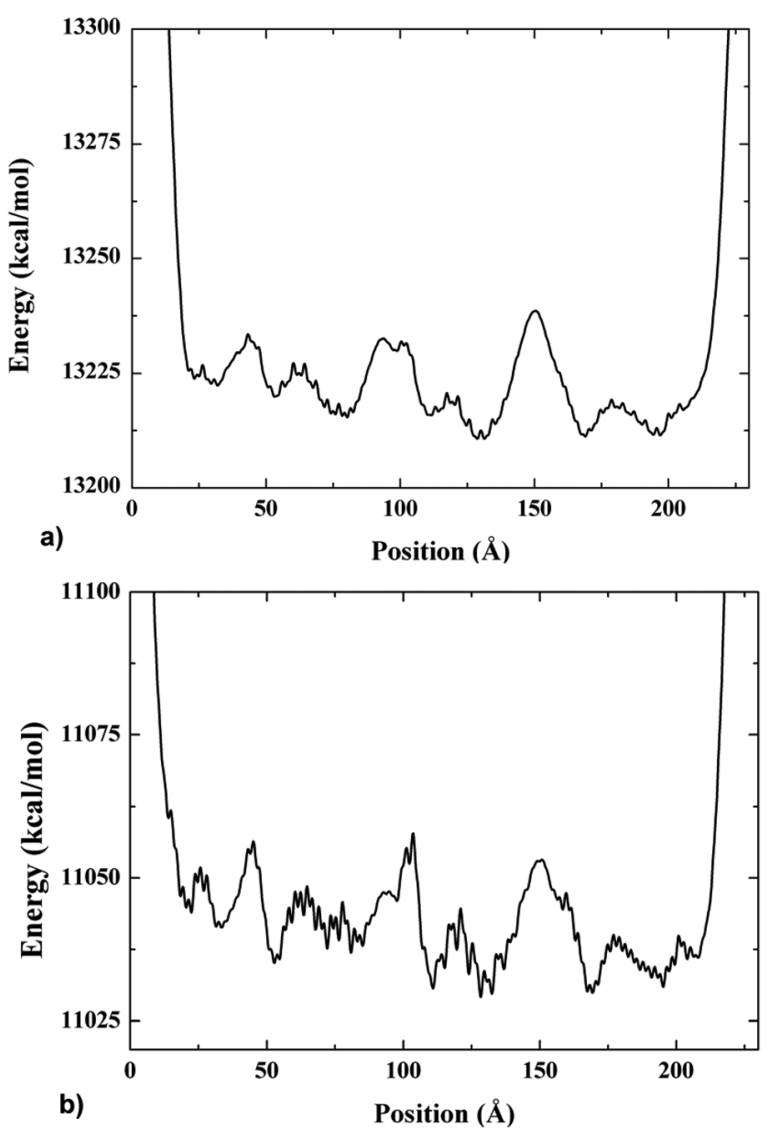

FIG. 4. Energy maps for the (a) graphene and (b) hBN. 


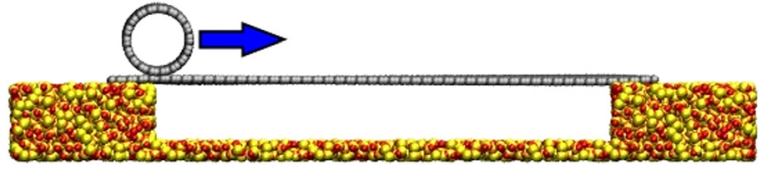

FIG. 5. Our proposed setup. The arrow indicates the direction in which we moved the CNT to compute the energy maps.

the sheet and the substrate which must be overcome. Thus, for the membrane to start curling the van der Waals attractive forces between it and the tube must become larger than the sum of its internal elastic forces and its van der Waals attraction to the substrate. The results reported by Zhang and $\mathrm{Li}^{13}$ showed it is indeed possible to overcome this attraction and then obtain a nanoscroll. However, our calculations strongly suggest this is a method of very low efficiency. In such a setup, not only the diameter of the CNT is of crucial importance, ${ }^{13}$ but we noticed that the initial position of the tube is also of great relevance. By moving the tube (in the sheet longitudinal direction) by distances as small as $0.5 \AA$, we can go from a situation in which a scroll is formed to one in which there is no formed scroll, which would render the method very inefficient.

In order to understand the origin of this significant dependence on small changes in the initial conditions for the scroll formation, we mapped the energy system configuration along the longitudinal direction of the sheet.
The maps were generated moving the CNT from one end to the other of the sheet and computing the system energy at each point. In Figure 4, we can see that there are various minima along the sheet length. What we observed is that for this method to yield a nanoscroll, two conditions must be satisfied: first the CNT must be initially positioned over a minimum position and second this minimum must be located close enough to the sheet edge. If one of these conditions is not satisfied, the decrease in energy due to the interaction between the membrane and the CNT will no longer be enough to overcome the substrate attraction and then the sheet will not be rolled up. Similar results were obtained for BN sheets (see Figure 4(b)).

Since these requirements pose serious limitations to the yield of self-formed scrolls, we devised an alternative approach. Instead of using a planar substrate configuration, we decided to try to use substrates containing chambers/pits on them, as shown in Figure 5. The length of this pit was of $180 \AA$ in our case. With this new configuration, the overall attraction between the sheet and the substrate would be reduced. It should be stressed that this kind of experimental setup, chambers/pits on silicon oxide substrates with graphene membranes deposited on them, has been already experimentally realized by many different groups. ${ }^{21}$ Thus, the proposed experimental setup is within our present day technology.

In Figure $6,{ }^{22}$ we present some representative snapshots from the molecular dynamics simulations using this new
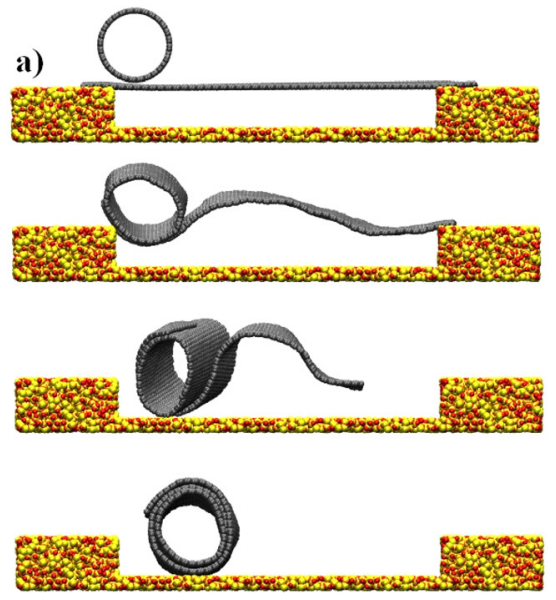

a)
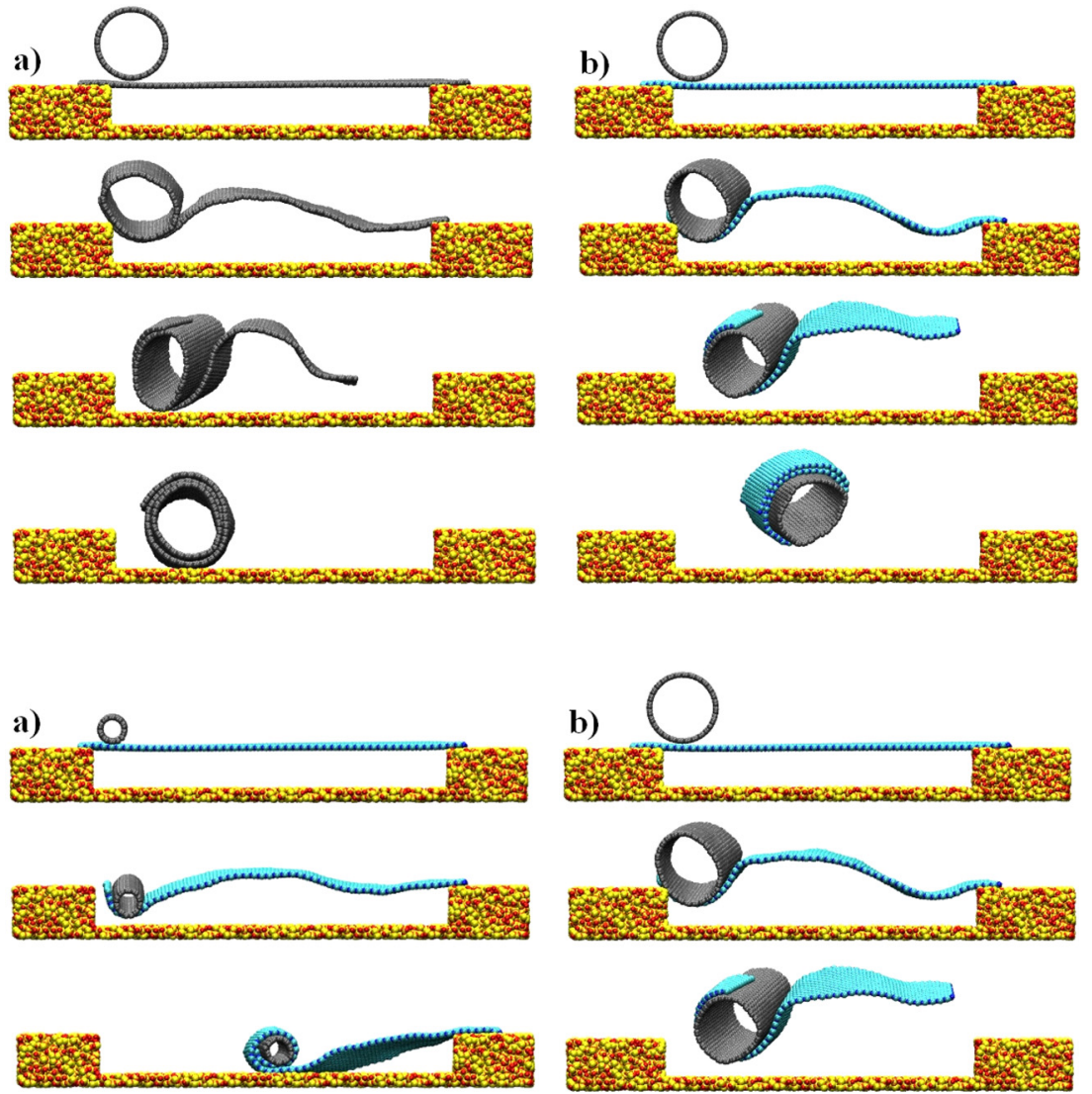

FIG. 6. Representative snapshots from the molecular dynamics simulations showing the scroll formation: (a) CNS and (b) BNNS.

FIG. 7. Representative snapshots from the molecular dynamics simulations showing the BNNS formation triggered by (a) $(10,10) \mathrm{CNT}$ and (b) $(28,28) \mathrm{CNT}$. 


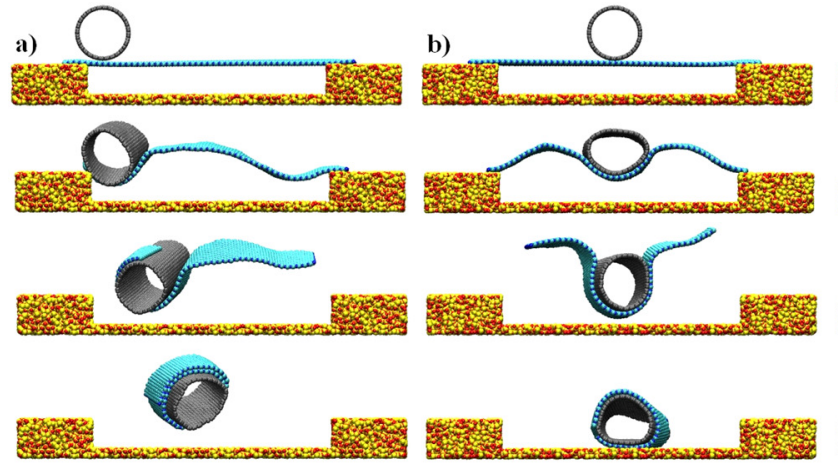

setup. Our results show that, using this approach, the scroll (for both, CNSs and BNNSs) formation yield significantly improved. From our simulations, we were able to obtain nanoscrolls by starting the process with nanotubes of a wide range of diameters, as shown in Figure $7,{ }^{22}$ and from many different initial positions, as shown in Figure $8 .^{22}$ In this setup, the tube diameter and the geometrical initial conditions are no longer limitations for the scroll formation, in contrast to the proposed setup by Zhang and $\mathrm{Li}^{13}$

In order to understand this significant increase in the nanoscroll formation efficiency, we mapped again the energy along the sheet and, as can be seen in Figure 9, the modified substrate completely changes the energy profile. Very deep minima can be seen on the edges of sheets and there is also a very wide and shallow minimum at the center. This renders

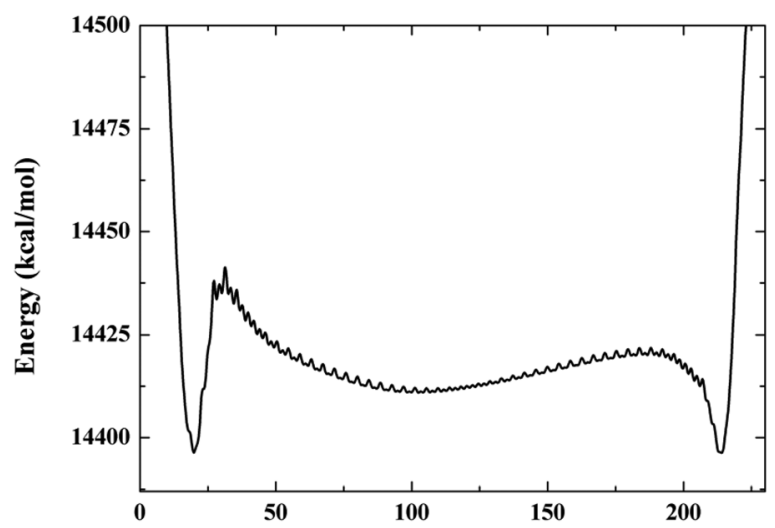

a)

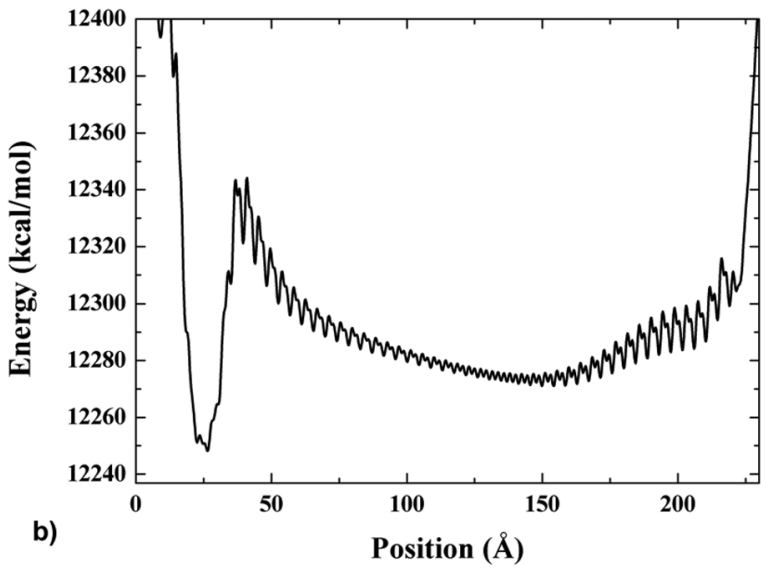

FIG. 9. Energy maps for the (a) graphene and (b) BN with our proposed setup.

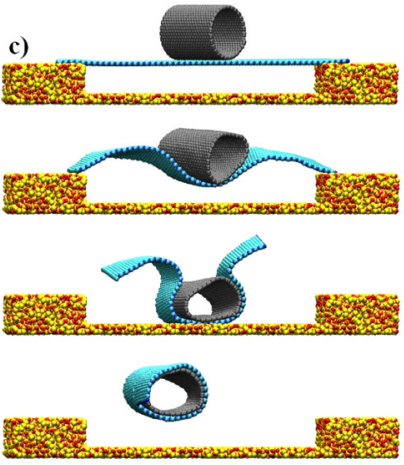

FIG. 8. Representative snapshots from the molecular dynamics simulations showing the BNNSs formation starting from different initial conditions. the small oscillations on the local energy completely irrelevant, making it possible to obtain nanoscrolls for a wide range of different initial conditions and making the process much more efficient.

Since this is a very simple setup in which we do not even need to have control over the CNT diameter or its fine placement position on the substrate, it appears to be a promising way to produce nanoscrolls. The nanoscroll dimensions can be tuned by the dimensions of the used membrane and, most importantly, this method is not only capable of yielding CNSs but is also the first proposed method for the production of single layer BNNSs. The BNNSs synthesis would represent a great advance on the field of boron nitride nanostructures, since it remains one of the few inorganic carbon equivalents that have not been realized yet.

\section{CONCLUSIONS}

In this work, we discussed a previously proposed method for producing carbon nanoscrolls and the origins of its limitations in terms of carbon nanotube diameter and its precise placement on the substrate. Based on that, we proposed an alternative setup (using substrates containing chambers/pits). This new setup proved to be more robust method and capable of overcoming some of the previous setup limitations, yielding nanoscrolls for a wide range of tube diameters and for several initial positions of the CNT used. Another important result is that, using the proposed method, it may be possible to produce not only CNSs but also other single layer scrolls from lamellar materials, like boron nitride.

After the submission of this manuscript, the first successful synthesis route to boron nitride nanoscrolls has been reported. $^{23}$

\section{ACKNOWLEDGMENTS}

We would like to thank Brazilian agencies Fundunesp, Fapesp, and CNPQ for financial support.

${ }^{1}$ R. Bacon, J. Appl. Phys. 31, 283 (1960).

${ }^{2}$ S. Ijima, Nature 354, 56 (1991).

${ }^{3}$ V. R. Coluci, S. F. Braga, R. H. Baughman, and D. S. Galvao, Phys. Rev. B 75, 125404 (2007).

${ }^{4}$ X. Shi, Y. Cheng, N. M. Pugno, and H. Gao, Appl. Phys. Lett. 96, 053115 (2010).

${ }^{5}$ M. L. Viculis, J. J. Mack, and R. B. Kaner, Science 299, 1361 (2003).

${ }^{6} \mathrm{H}$. Shioyama and T. Akita, Carbon 41, 179 (2003). 
${ }^{7}$ D. Tomanek, Physica B 323, 86 (2002).

${ }^{8}$ S. F. Braga, V. R. Coluci, S. B. Legoas, R. Giro, D. S. Galvao, and R. H. Baughman, Nano Lett. 4, 881 (2004).

${ }^{9}$ H. Pan, Y. Feng, and J. Lin, Phys. Rev. B 72, 085415 (2005).

${ }^{10}$ R. Rurali, V. R. Coluci, and D. S. Galvao, Phys. Rev. B 74, 085414 (2006).

${ }^{11}$ V. M. Savoskin, V. N. Mochalin, A. P. Yaroshenki, N. I. Lazareva, T. E. Konstantinova, I. V. Barsukov, and I. O. Prokofiev, Carbon 45, 2797 (2007).

${ }^{12}$ X. Xie, L. Ju, X. Feng, Y. Sun, R. Zhou, K. Liu, S. Fan, Q. Li, and K. Jiang, Nano Lett. 9, 2565 (2009).

${ }^{13}$ Z. Zhang and T. Li, Appl. Phys. Lett. 97, 081909 (2010).

${ }^{14}$ L. Chu, Q. Xue, T. Zhang, and C. Ling, J. Phys. Chem. C 115, 15217 (2011).

${ }^{15}$ X. Chen, L. Li, X. Sun, H. G. Kia, and H. Peng, Nanotechnology 23, 055603 (2012).
${ }^{16}$ E. Perim and D. S. Galvão, Nanotechnology 20, 335702 (2009).

${ }^{17}$ A. Rubio, J. L. Corkill, and M. L. Cohen, Phys. Rev. B 49, 5081 (1994).

${ }^{18}$ X. Blasé, A. Rubio, S. G. Louie, and M. L. Cohen, Europhys. Lett. 28, 335 (1994).

${ }^{19}$ A. K. Rappe, C. J. Casewit, K. S. Colwell, W. A. Goddard III, and W. M. Skiff, J. Am. Chem. Soc. 114, 10024 (1992).

${ }^{20}$ MATERIALS STUDIO is a suite of simulation programs available from Accelrys. Accelrys, Inc. 10188 Telesis Court, Suite 100, San Diego, CA, 9212, USA, http://www.accelrys.com.

${ }^{21}$ J. S. Bunch et al., Nano Lett. 8, 2458 (2008).

${ }^{22}$ See supplementary material at http://dx.doi.org/10.1063/1.4790304 for corresponding videos of Figs. 6-8, where these processes can be better visualized.

${ }^{23}$ X. Chen, R. A. Boulos, J. F. Dobson, and C. L. Raston, Nanoscale 5, 498 (2013). 